\title{
TESTIMONIOS
}

\section{APUNTES DE MEMORIA}

\author{
MEMORY NOTES
}

Doroteo Arnáiz

Artista

El testimonio de unos hechos ocurridos hace cincuenta años, sostenido con el recurso primordial de los recuerdos y de algunos documentos originales, guardados para mantener dichos recuerdos en constante re-actualización, permite abordar una situación de diálogo con un lector que espera recibir una información lo más veraz posible, de «primera mano» y despojada de medio siglo de subjetividades y medias-verdades que ya se han instalado en la Historia con mayúscula.

Inscrito en la Escuela Nacional Superior de Bellas Artes de París (ENSBA), desde 1960, en las clases de los profesores Robert Cami y Jean E. Bersier, en mayo del 68 me encontraba en el taller de talla-dulce trabajando una plancha, la última que realizaría en dicho taller, cuando los acontecimientos ya conocidos me sorprendieron, incluyéndome en la intensa actividad que en aquellos locales iba a desarrollarse.

Si nos preguntamos cómo se gestó el Atelier Populaire de la Escuela, tenemos una posible respuesta: la reputación de la Escuela, como centro de enseñanza superior clásica y «libre» al mismo tiempo, era conocida en Francia y allende sus fronteras. Por los talleres de pintura, escultura, grabado y otras técnicas, habían pasado numerosos artistas que gozaban de suficiente notoriedad tanto en Francia como en otros países de origen $y$, entre aquellos que trabajaban en París, muchos no eran ajenos a los acontecimientos que se estaban produciendo.

En alguna de las asambleas que se estaban celebrando en diversos centros de la capital alguien propuso imprimir carteles y la lógica casualidad puso el foco en la Escuela. Allí se presentó un grupo de personas, incluyendo estudiantes de otras facultades, acompañados de algunos artistas que se instalan en el taller de Maurice Brianchon. Seguidamente, tras convenir las fórmulas de trabajo, suben a los talleres de grabado y litografía para ocuparlos y dedicarse a imprimir los carteles. Los estudiantes que se encuentran trabajando tratan de convencerlos de que aquellos sistemas de estampación son inoperantes para la cantidad 
de carteles que se querían imprimir. Después de una tentativa con la litografía, de tensos diálogos y algunas idas y venidas al taller Brianchon, se resuelve adoptar la serigrafía como la técnica manual más apropiada para desarrollar una buena cadencia de impresión. Posteriormente, se conseguirían imprentas que pondrían la técnica del offset a disposición de otras facultades y talleres.

En los primeros días de producción de carteles, pasaron muchas personas conocidas por la Escuela, entre otras Pierre Gaudibert, conservador del Museo de Arte Moderno de la Villa de París y promotor de ARC -Art, Recherche et Confrontation-, con quien mantenía una cordial relación. Charlando con él, convinimos en la oportunidad que se presentaba de aprovechar la situación de la Escuela como punto de encuentro para agrupar a aquellos artistas que llevábamos mucho tiempo reclamando una serie de derechos sociales. Inmediatamente, fueron apareciendo artistas que lo habían contactado.

La primera reunión contó con la participación del propio Gaudibert, Henri Cueco y Jean de Gaspary, entre otros; después llegaron más participantes, algunos asistían a dos o tres reuniones y luego no volvían. Sin embargo, la afluencia de artistas fue creciendo hasta concluir en lo que se denominó el MIRA -Mouvement International Révolutionnaire des Arts (Movimiento Internacional Revolucionario de las Artes)-, cuyos postulados fueron comunicados en un manifiesto. Las banderolas del MIRA estuvieron presentes en diversos actos y manifestaciones sostenidas por los esforzados Yannick Ballif y Jean Hubert Attali. Cuando llegó la transformación de este movimiento en FAP -Front des Artistes Plasticiens (Frente de los Artistas Plásticos)-, que reunió a diversos grupos de distintas ideologías, las exigencias del MIRA estuvieron presentes en los encuentros con los responsables de la Maison des Artistes, para acordar la fórmula de admisión de todas las profesiones de las artes plásticas al régimen general de la Seguridad Social y el acceso a los talleres de alquiler moderado, incluyendo los artistas extranjeros residentes en Francia.

Mi relación con la Escuela no concluyó el día que la policía cerró los talleres de grabado, vaciando todos los materiales que allí se encontraban. Poco tiempo después fui invitado anualmente por el consejo pedagógico, en calidad de miembro externo del jurado de la sección de pintura, en la prueba final del diploma, desde 1972 hasta 1981.

Mucho se ha escrito en este medio siglo pasado sobre lo útil o estéril que fueron las revueltas iniciadas un 22 de marzo, lo que sí se puede decir es que muchas cosas cambiaron y tal vez la mejor forma de concluir es utilizando una frase del periodista Lance Morrow: «1968 separó como un cuchillo el pasado del futuro».

Doroteo Arnáiz, 15 de agosto de 2018. 
Ce qui nous a réunis :

1) Nos activités révolutionnaires passées.

Nos rencontres dans l'action de ces jours derniers.

La cohabitation féconde de nos accords et de nos divergences.

2) Divergence des courants de pensée et de nos conceptions esthétiques.

Convergences de notre pensée politique.

3) Besoin de participation profonde à la pensée et l'action politique.

Ne plus être des instruments d'une ornementation de classe.

Assurer la liberté de l'imagination sans laquelle toute pensée ne peut être féconde.

4) Nous croyons nécessaire en conséquence de tracer les lignes de force d'une culture révolutionnaire. Ce qui suppose une remise en question de tout le système étatique, marchand, et de toute idéologie de l'art.

5) L'objectif idéal de l'action révolutionnaire est d'assurer la liberté de l'homme, de tous les hommes.

L'expression de cette liberté est la suppression de l'autorité plus ou moins répressive de tout État, c'est-à-dire une société d'adultes libres et responsables.

Nous pensons que l'action révolutionnaire profonde suppose chez les individus et les groupes cette motivation consciente ou non, affective ou rationnalisée.

6) Nous croyons que cohabitent en chacun de nous le révolté et révolutionnaire et qu'il n'est pas possible de choisir l'un au détriment de l'autre ou d'étouffer l'un par l'autre; nous pensons que les deux pôles de la pensée révolutionnaire doivent exister, en tension, à l'intérieur de chacun de nous et que l'action révolutionnaire doit être féconde par cette échange et par cette tension.

MIRA $^{2}$

Lo que nos ha reunido:

1) Nuestras actividades revolucionarias pasadas.

Nuestros encuentros en la acción de estos últimos días.

La cohabitación fecunda de nuestros acuerdos y de nuestras divergencias.

1 Documentos originales aportados por el artista Doroteo Arnáiz. En primer lugar, aparecen las notas tomadas por el propio Arnáiz en una de las primeras reuniones del grupo y, en segundo lugar, el manifiesto del MIRA.

2 Todas las traducciones son de Patricia Badenes Salazar. 
2) Divergencia de las corrientes de pensamiento y de nuestras concepciones estéticas.

Convergencias de nuestro pensamiento político.

3) Necesidad de participación profunda en el pensamiento y la acción política.

No ser más instrumentos de una ornamentación de clase.

Asegurar la libertad de la imaginación sin la cual ningún pensamiento puede ser fecundo.

4) Creemos necesario, en consecuencia, trazar las líneas fuertes de una cultura revolucionaria. Lo que supone una puesta en entredicho de todo el sistema estatal, comercial, y de toda ideología del arte.

5) El objetivo ideal de la acción revolucionaria es asegurar la libertad del hombre, de todos los hombres.

La expresión de esta libertad es la supresión de la autoridad, más o menos represiva, de todo el Estado, es decir, una sociedad de adultos libres y responsables.

Pensamos que la acción revolucionaria profunda supone para los individuos y los grupos esta motivación consciente o no, afectiva o racionalizada.

6) Creemos que cohabitan en cada uno de nosotros el rebelde y el revolucionario y que no es posible elegir uno en detrimento del otro o ahogar uno en el otro; pensamos que los dos polos del pensamiento revolucionario deben existir, en tensión, en el interior de cada uno de nosotros y que la acción revolucionaria debe ser fecunda gracias a ese intercambio y a dicha tensión.

\section{Mouvement International Révolutionnaire des Arts MANIFESTE}

L'art ne doit pas être le privilège de quelques uns mais doit au contraire être mis à la disposition de tous. Dans toute société de classe, la situation de l'artiste risque de tourner à l'aliénation, aliénation économique d'une part résultant de ce que l'activité artistique étant encore considérée comme une activité non strictement nécessaire à la société, l'œuvre d'art ne peut appartenir à l'heure actuelle qu'aux groupes disposant de revenus suffisants pour effectuer l'achat d'objets "de luxe », aliénation spirituelle d'autre part en ce que les valeurs artistiques se sont élaborées à partir de valeurs culturelles marquées par la domination d'une classe sur l'activité intellectuelle des siècles passés.

Pour que l'art puisse effectivement être mis à la disposition de tous il est donc nécessaire que le cadre à l'intérieur duquel les contacts entre artiste et public s'établissent, dépasse le niveau d'un groupe économiquement et culturellement repérable. 
Ceci suppose qu'une action soit entreprise tant au niveau économique par la mise à la portée de l'œuvre d'art des classes non possédantes, qu'au niveau culturel par la remise en question pratique des valeurs esthétiques et culturelles définissant le groupe de référence de l'artiste.

Cette remise en question ne peut se faire qu'à partir d'une ouverture de l'artiste vers tous ${ }^{3}$ les groupes composant la société à l'intérieur de laquelle il vit, ouverture qui se traduira par un effort de contact menant par la force même des choses à la confrontation et au dialogue, ce qui permettra à l'artiste de renouer avec les liens lui permettant de s'exprimer en fonction d'une conscience qui soit proprement collective.

Le Mira se propose donc de mettre sur pied une organisation facilitant les rapports entre l'artiste et l'ensemble effectif de la société. II en attend l'apparition de nouvelles structures mentales et d'une nouvelle forme de dialogue permettant à l'art menacé de sclérose de redevenir une activité proprement vivante exprimant les valeurs, les angoisses et les joies non plus d'une classe particulière mais d'une société.

Considérant que c'est du foisonnement, même désordonné des idées et des tentatives artistiques que peuvent surgir les nouvelles formes d'art et de contact qu'il recherche, le Mira pose le principe du refus de toute forme de discrimination à l'égard des artistes qui voudraient le rejoindre. En particulier le Mira tient à affirmer qu'il refuse absolument toute forme de sélection reposant sur des bases esthétiques préétablies car il estime que toute forme de sélection ne peut résulter que des structures mentales et des valeurs esthétiques et culturelles existantes à l'heure actuelle.

L'adoption de tout critère sélectif ne pourrait donc en définitive que freiner le jaillissement des idées au nom d'une orthodoxie esthétique et mènerait donc à la constitution de chapelles qui s'opposeraient à l'émergence d'un art libre et vivant.

En conséquence le présent manifeste refuse qu'un artiste même tenu pour génial puisse jamais être considéré comme représentatif $d$ 'autre chose que d'une esthétique péronnelle et donc puisse jamais être considéré comme un interlocuteur valable en ce qui concerne la définition de l'ensemble des rapports artiste-public.

Enfin, par les principes mêmes sur lesquels il entend faire reposer son action, le Mira affirme sa vocation à l'universalité d'adhésion. 
Le Mira ne veut se rattacher à aucune orthodoxie politique. Les principes sur lesquels repose son action lui imposent néanmoins de définir un certain nombre d'idées directrices concernant la façon dont il entend s'intégrer à la société. Ces idées directrices du fait même qu'elles touchent au problème de l'intégration de l'artiste dans le groupe, seront, de par leur nature, politiques. Le but même qu'il s'est fixé oblige le mouvement à se définir par rapport aux institutions et aux structures mentales existantes. De la définition des principes auxquels doivent obéir ces rapports découle une ligne politique. Sans vouloir rappeler ici les liens qui existent entre la forme que doit prendre une organisation et les principes qui guident son action, d'une part, et les buts que se fixe cette organisation d'autre part, le Mira réaffirme ici que toute faiblisse sur le plan des principes ne peut qu'entrainer la dégénérescence, la compromission, ou la disparition du mouvement.

En conséquence le Mira tient à préciser ici l'attitude qu'il entend observer à l'égard des institutions et des organisations existantes d'une part, à l'égard du public de l'autre.

En ce qui concerne les rapports à entretenir avec les institutions et les organisations existantes, le présent manifeste s'inspire du principe de résistance aux organisations et aux institutions; principe basé sur la constatation de résultats auxquels la collaboration avec ces institutions ne manqueraient pas de mener, c'est-à-dire à l'absorption et à l'intégration du mouvement aux structures existantes. La preuve ayant été faite maintes et maintes fois que les réformes de l'intérieur ne peuvent quand elles s'attaquent à des structures solidement établies et soutenues qu'aboutir à la collaboration de fait et à la sclérose du mouvement. Le Mira adopte le principe de refus de collaboration avec les institutions qui, par leur forme ou leur esprit risquent de prolonger et de fortifier la situation existante, le principe de parallélisme à ces institutions et le principe d'agressivité à leur égard.

En ce qui concerne les rapports avec le public, le Mira refuse le point de vue selon lequel il existe une partie «cultivée » de la population à laquelle l'œuvre d'art s'adresse par "vocation », mais constate que le sentiment artistique étant inné chez l'homme la distinction régnant à l'heure actuelle résulte d'un blocage de type psychologique et social. II se refuse donc à accepter et à entériner par son action le prétendu statu quo de l'artiste et du public. II affirme au contraire qu'une action immédiate peut efficacement contribuer à la destruction de ce blocage mental. Cette action ne peut à ses yeux reposer que sur le principe de familiarisation avec l'œuvre d'art, suivie ou doublée d'un effort tendant à instaurer la contestation de l'artiste et le dialogue.

II refuse donc en conséquence toute action inspirée par une politique du type « création de besoin " dans une optique de capitalisme publicitaire, toute action reposant sur ces bases ne pouvant entrainer qu'une mode qui $d^{\prime}$ 'une part ne serait que passagère et $d^{\prime}$ autre 
part ne reflèterait les besoins artistiques du public que par l'intermédiaire d'un snobisme superficiel ou d'un engovement éphémère compromettant ainsi profondément toutes chances de dialogue réel.

Au contraire le Mira prétend appuyer son action sur le principe de RÉVÉLATION des besoins par la destruction d'inhibitions sociales. De ce principe découlent trois idées directrices:

Primo, la destruction de l'inhibition sociale passe par la connaissance du monde de l'esthétique, connaissance qui ne peut se faire que par la familiarisation progressive du nouveau public avec les œuvres d'art.

Secundo, la destruction de l'inhibition sociale passe par une redéfinition des rapports existants entre le public et l'œuvre d'art. En particulier il est nécessaire que l'idée d'œuvre sacralisée facteur de valeurs « culturelles » disparaisse tant que le concept de culture restera entaché de déterminismes sociaux. Ceci suppose que la familiarisation se fasse au niveau de la communion et de la perception immédiates de l'œuvre d'art, et non plus par le biais de l'accession à un monde culturel en soi. De cette nécessité découlera une certaine conception de la forme même que doivent prendre les expositions et les contacts.

Tertio enfin, la destruction de l'inhibition sociale passe par l'établissement d'un contact entre le nouveau public et l'artiste, contact permettant au nouveau public de voir s'exprimer ses propres aspirations et son propre monde de référence dans l'œuvre d'art. II d'agit ici de détruire le privilège de fait dont jouit la partie dite cultivée de la société d'entrer en contact avec l'artiste, et d'orienter donc la recherche de l'artiste dans une direction qui reflète les aspirations de ce groupe social déterminé.

Tant que le public ne pourra pas entrer en contact direct avec l'artiste, l'artiste ne pourra ressentir les aspirations du public. Tant que l'artiste ne pourra ressentir ces aspirations là, le nouveau public ne pourra se reconnaitre dans les œuvres qu'on lui propose.

De l'application de ces principes le Mira attend la transformation des rapports existants entre l'artiste et le nouveau public. Ces rapports sont à l'heure actuelle enfermés dans l'alternative acceptation-refus. Le but est de la faire évolver vers l'alternative acceptationdialogue, ce qui suppose une première phase acceptation-contestation. Ceci ne pourra se faire que si l'artiste arrive effectivement à entretenir avec le nouveau public un rapport vivant et intéressé.

Le Mira s'engage à appliquer les principes contenus dans ce manifeste au niveau de son action pratique à laquelle il veut associer tous les artistes prêts à accepter pleinement les principes ci-énoncés.

Paris, le 7 juin 1968. 


\section{Movimiento Internacional Revolucionario de las Artes MANIFIESTO}

El arte no debe ser el privilegio de unos pocos, debe, al contrario, ser puesto a disposición de todos. En toda sociedad de clase, la situación del artista corre el riesgo de desembocar en la alienación; alienación económica, de una parte, como consecuencia de que la actividad artística estando todavía considerada como una actividad no estrictamente necesaria para la sociedad, la obra de arte sólo puede pertenecer, en el momento actual, a los grupos que disponen de ingresos suficientes para efectuar la compra de objetos de «lujo»; alienación espiritual, de otra parte, en cuanto que los valores artísticos son elaborados a partir de valores culturales marcados por la dominación de una clase sobre la actividad intelectual de los siglos pasados.

Para que el arte pueda efectivamente ser puesto a disposición de todos es necesario pues que el marco en cuyo interior los contactos entre artista y público se establecen, supere el nivel de un grupo económica y culturalmente localizable.

Esto implica que una acción sea emprendida tanto a nivel económico, a través del acercamiento de la obra de arte a las clases desposeídas, como a nivel cultural, a través de la puesta en duda práctica de los valores estéticos y culturales que definen el grupo de referencia del artista.

Esta puesta en entredicho sólo puede hacerse a partir de una apertura del artista hacia todos los grupos que componen la sociedad donde él vive, apertura que se traducirá en un esfuerzo de contacto que lleva, por la fuerza misma de las cosas, a la confrontación y al diálogo, lo que permitirá al artista reconectar con los vínculos que le llevan a expresarse en función de una conciencia que sea propiamente colectiva.

El Mira se propone pues poner en marcha una organización que facilite las relaciones entre el artista y el conjunto efectivo de la sociedad. Espera la aparición de nuevas estructuras mentales y de una nueva forma de diálogo que permita al arte amenazado de esclerosis volver a convertirse en una actividad propiamente viva que exprese los valores, las angustias y las alegrías no sólo de una clase particular sino de una sociedad.

Considerando que es de la afluencia, incluso desordenada, de las ideas y de las tentativas artísticas que pueden surgir las nuevas formas de arte y de contacto que busca, el Mira plantea el principio del rechazo de toda forma de discriminación con respecto a los artistas que querrían unírsele. En particular, el Mira quiere afirmar que rechaza completamente toda forma de selección que descanse sobre unas bases estéticas preestablecidas ya que 
considera que toda forma de selección sólo puede provenir de las estructuras mentales y de los valores estéticos y culturales existentes en la hora actual.

La adopción de todo criterio selectivo sólo podría pues, en definitiva, frenar la erupción de las ideas en nombre de una ortodoxia estética y conduciría así a la constitución de capillas que se opondrían al surgimiento de un arte libre y vivo.

En consecuencia, el presente manifiesto rechaza que un artista, incluso calificado de genial, pueda jamás ser considerado como representativo de otra cosa que una estética parlanchina $y$, por lo tanto, pueda jamás ser tenido como un interlocutor válido en lo que concierne a la definición del conjunto de relaciones artista-público.

En fin, por los principios mismos sobre los cuales exige hacer descansar su acción, el Mira afirma su vocación de universalidad de adhesión.

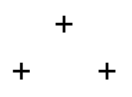

El Mira no quiere vincularse a ninguna ortodoxia política. Los principios sobre los cuales reposa su acción le imponen, sin embargo, definir un cierto número de ideas directrices que conciernen la manera en la que exige integrarse en la sociedad. Estas ideas directrices, en la medida en que abordan el problema de la integración del artista en el grupo, serán, por su naturaleza, políticas. El propio objetivo que se ha fijado obliga al movimiento a definirse en relación a las instituciones y a las estructuras mentales existentes. De la definición de los principios a los que deben obedecer estas relaciones se deriva una línea política. Sin querer recordar aquí los lazos que existen entre la forma que debe tomar una organización y los principios que guían su acción, por un lado, y los objetivos que se fija esta organización, por otro, el Mira reafirma aquí que toda debilidad en el plano de los principios sólo puede comportar la decadencia, el acomodo o la desaparición del movimiento.

En consecuencia, el Mira quiere precisar aquí la actitud que exige tener de cara a las instituciones y a las organizaciones existentes, de una parte; de cara al público, de otra.

Por lo que concierne a las relaciones que mantiene con las instituciones y las organizaciones existentes, el presente manifiesto se inspira en el principio de resistencia frente a las organizaciones y a las instituciones; principio basado en la constatación de resultados a los que la colaboración con estas instituciones no evitaría conducir, es decir, a la absorción y a la integración del movimiento en las estructuras existentes. Habiendo sido hecha muchas, muchas, veces la prueba, las reformas del interior sólo pueden, cuando ellas atacan a estructuras sólidamente establecidas y sostenidas, desembocar en la colaboración de hecho y a la esclerosis del 
movimiento. El Mira adopta el principio de rechazo de colaboración con las instituciones que, por su forma o su espíritu, pueden prolongar y fortalecer la situación existente, el principio de paralelismo hacia estas instituciones y el principio de agresividad de cara a ellas.

En cuanto a las relaciones con el público, el Mira rechaza el punto de vista según el cual existe una parte "cultivada» de la población a la que se dirige la obra de arte por «vocación», pero constata que el sentimiento artístico, siendo innato en el hombre, la distinción reinando actualmente, resulta de un bloqueo de tipo psicológico y social. Se niega pues a aceptar y a ratificar por su acción el statu quo del artista y del público. Afirma, al contrario, que una acción inmediata puede, de manera eficaz, contribuir a la destrucción de este bloqueo mental. Esta acción sólo puede, a sus ojos, descansar sobre el principio de familiarización con la obra de arte, seguida o redoblada de un esfuerzo tendente a instaurar la contestación del artista y el diálogo.

Rechaza, pues, en consecuencia, toda acción inspirada por una política del tipo «creación de necesidad» en una óptica de capitalismo publicitario, toda acción que repose sobre estas bases sólo puede conllevar una moda que, por un lado, sólo sería pasajera y, por otro, sólo reflejaría las necesidades artísticas del público a través del intermediario de un esnobismo superficial o de un entusiasmo efímero que compromete así, profundamente, todas las oportunidades de diálogo real.

Al contrario, el Mira pretende apoyar su acción sobre el principio de REVELACIÓN de las necesidades a través de la destrucción de inhibiciones sociales. De este principio derivan tres ideas directrices:

Primero, la destrucción de la inhibición social pasa por el conocimiento del mundo de la estética, conocimiento que sólo puede hacerse a través de la familiarización progresiva del nuevo público con las obras de arte.

Segundo, la destrucción de la inhibición social pasa por una redefinición de las relaciones existentes entre el público y la obra de arte. En particular, es necesario que la idea de obra sacralizada, factor de valores «culturales», desaparezca tanto en cuanto el concepto de cultura permanecerá manchado por determinismos sociales. Esto supone que la familiarización se haga a nivel de la comunión y de la percepción inmediatas de la obra de arte, y no por la vía de la adhesión a un mundo cultural en sí. De esta necesidad surgirá una cierta concepción de la forma misma que deben tomar las exposiciones y los contactos.

Tercero, en fin, la destrucción de la inhibición social pasa por el establecimiento de un contacto entre el nuevo público y el artista, contacto que permite al nuevo público ver expresarse sus propias aspiraciones y su propio mundo de referencia en la obra de arte. Se trata aquí de destruir el privilegio de hecho, del que disfruta la parte llamada cultivada de la 
sociedad, de entrar en contacto con el artista, y de orientar, pues, la búsqueda del artista en una dirección que refleja las aspiraciones de este grupo social determinado.

En tanto en cuanto el público no podrá entrar en contacto directo con el artista, el artista no podrá experimentar las aspiraciones del público. En tanto en cuanto el artista no podrá experimentar estas aspiraciones, el nuevo público no podrá reconocerse en las obras que se le proponen.

De la aplicación de estos principios, el Mira espera la transformación de las relaciones existentes entre el artista y el nuevo público. Estas relaciones están, en este momento, encerradas en la alternativa aceptación-rechazo. El objetivo es hacerla evolucionar hacia la alternativa aceptación-diálogo, lo que supone una primera fase aceptación-contestación. Esto sólo podrá hacerse si el artista llega, efectivamente, a mantener con el nuevo público una relación viva e interesada.

El Mira se compromete a aplicar los principios contenidos en este manifiesto a nivel de su acción práctica a la cual quiere asociar a todos los artistas preparados para aceptar, plenamente, los principios aquí anunciados.

París, 7 de junio de 1968.

Al hilo de los sucesos de Mayo, un grupo de artistas plásticos decidieron contribuir a la causa del movimiento estudiantil y obrero y crear un comité de acción como los que en aquellos días surgían por doquier. Así nació Le Comité d'Action Arts Plastiques, cuyos principales postulados recogieron en un breve texto fundacional. He aquí la transcripción íntegra del documento; unas escuetas líneas que reflejan a la perfección el latido de la época y el pulso de sus ideas ${ }^{4}$ :

LE COMITÉ D'ACTION ARTS PLASTIQUES met en garde contre la multiplication $d^{\prime}$ initiatives qui resteraient dispersées en tous sens, alors que la situation et la lutte en cours exigent des actions qui engagent la responsabilité de tous.

Il déclare en outre que la majorité des artistes présents au Forum de l'Institut d'Art et $d$ 'Archéologie ont envisagé différentes formes d'action pour participer au mouvement des étudiants et des ouvriers parmi lesquelles:

4 Doroteo Arnáiz no formó parte de este comité, pero conserva y nos ha dado a conocer este interesante documento, testimonio valiosísimo de una época. 
- Le boycott des Institutions et manifestations culturelles symboles du pouvoir politique actuel et de la politique de prestige de ce même pouvoir.

- La contestation des structures socio-économiques de distribution de l'Art.

Sur le point particulier immédiat de l'action politique des galeries deux possibilités nous semblent pouvoir être discutés par les galeries elles-mêmes:

- Soit fermeture de la galerie avec une affiche apposée indiquant «Solidarité avec le mouvement étudiant et ouvrier».

- Soit transformation de la galerie en centre d'opposition politique par des affiches, l'exposition de documents, de photographies, ou œuvres en rapport avec la lutte, la collecte d'argent pour le mouvement, etc., etc.

Le Comité d'Action d'Arts Plastiques.

EL COMITÉ DE ACCIÓN ARTES PLÁSTICAS advierte contra la multiplicación de iniciativas que permanecerían dispersas en todas las direcciones, mientras que la situación y la lucha en curso exigen acciones que impliquen la responsabilidad de todos.

Asimismo, declara que la mayoría de los artistas presentes en el Fórum del Instituto de Arte y de Arqueología han previsto diferentes formas de acción para participar en el movimiento de los estudiantes y de los obreros, entre otras:

-El boicot a las instituciones y a las manifestaciones culturales símbolos del poder político actual y de la política de prestigio de este mismo poder.

- La contestación de las estructuras socioeconómicas de distribución del Arte.

Sobre el punto particular inmediato de la acción política de las galerías, dos posibilidades nos parecen poder ser discutidas por las propias galerías:

- Sea el cierre de la galería con un cartel colgado que indique "Solidaridad con el movimiento estudiantil y obrero".

- Sea la transformación de la galería en un centro de oposición política a través de los afiches, la exhibición de documentos, de fotografías, u obras relacionadas con la lucha, la colecta de dinero para el movimiento, etc., etc. .........

El Comité de Acción de Artes Plásticas.

Dossiers Feministes, 24, 2018, 125-144 - ISSN: $1139-1219$ - DOI: http://dx.doi.org/10.6035/Dossiers.2018.24.9 
Doroteo Arnáiz guarda celosamente ocho afiches que logró salvar de las destructivas «llamas» de la vuelta al orden. Rescatados in extremis del Atelier Populaire, donde él estuvo colaborando en su elaboración, he aquí una reproducción de esos magníficos carteles.

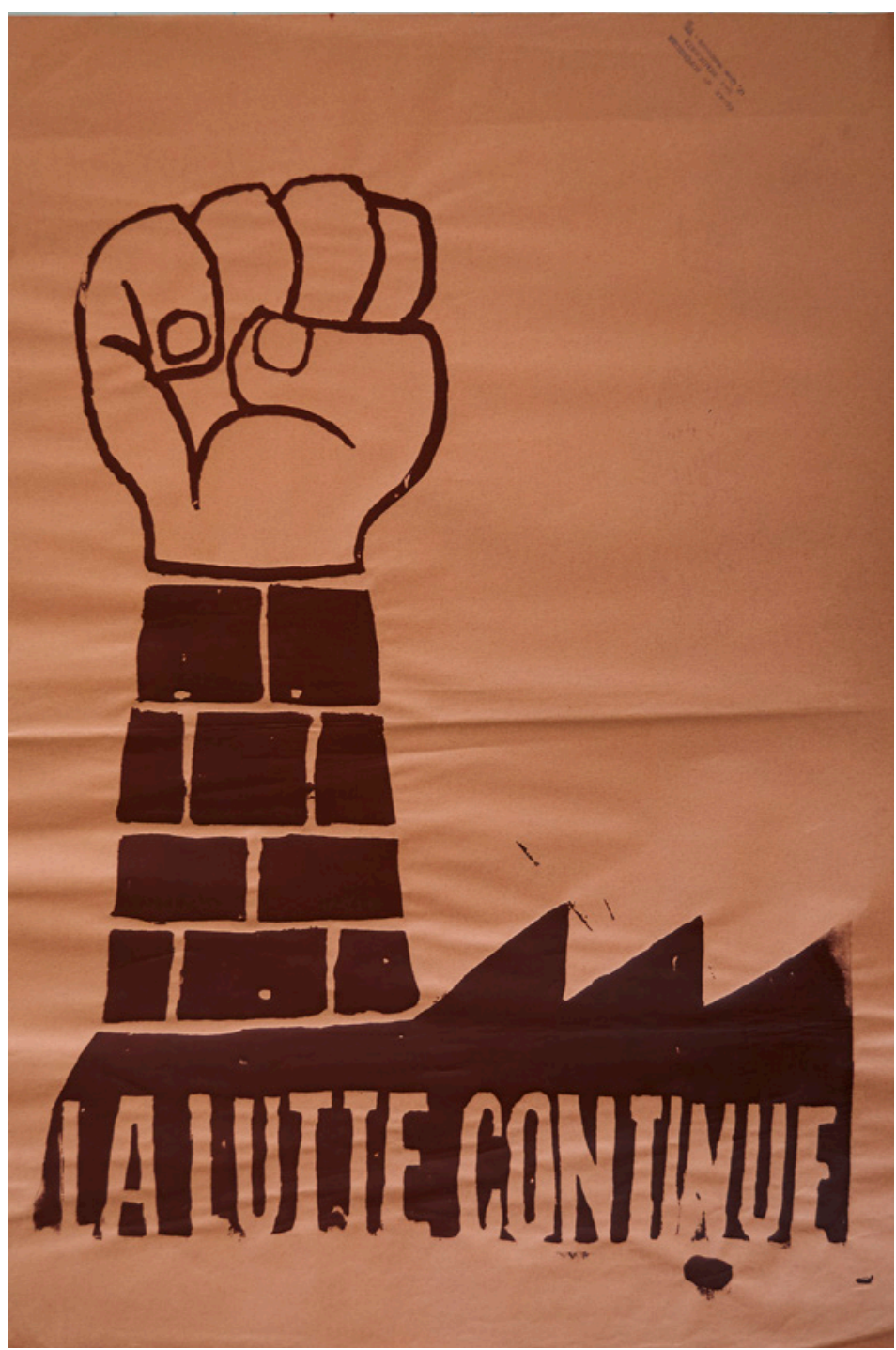




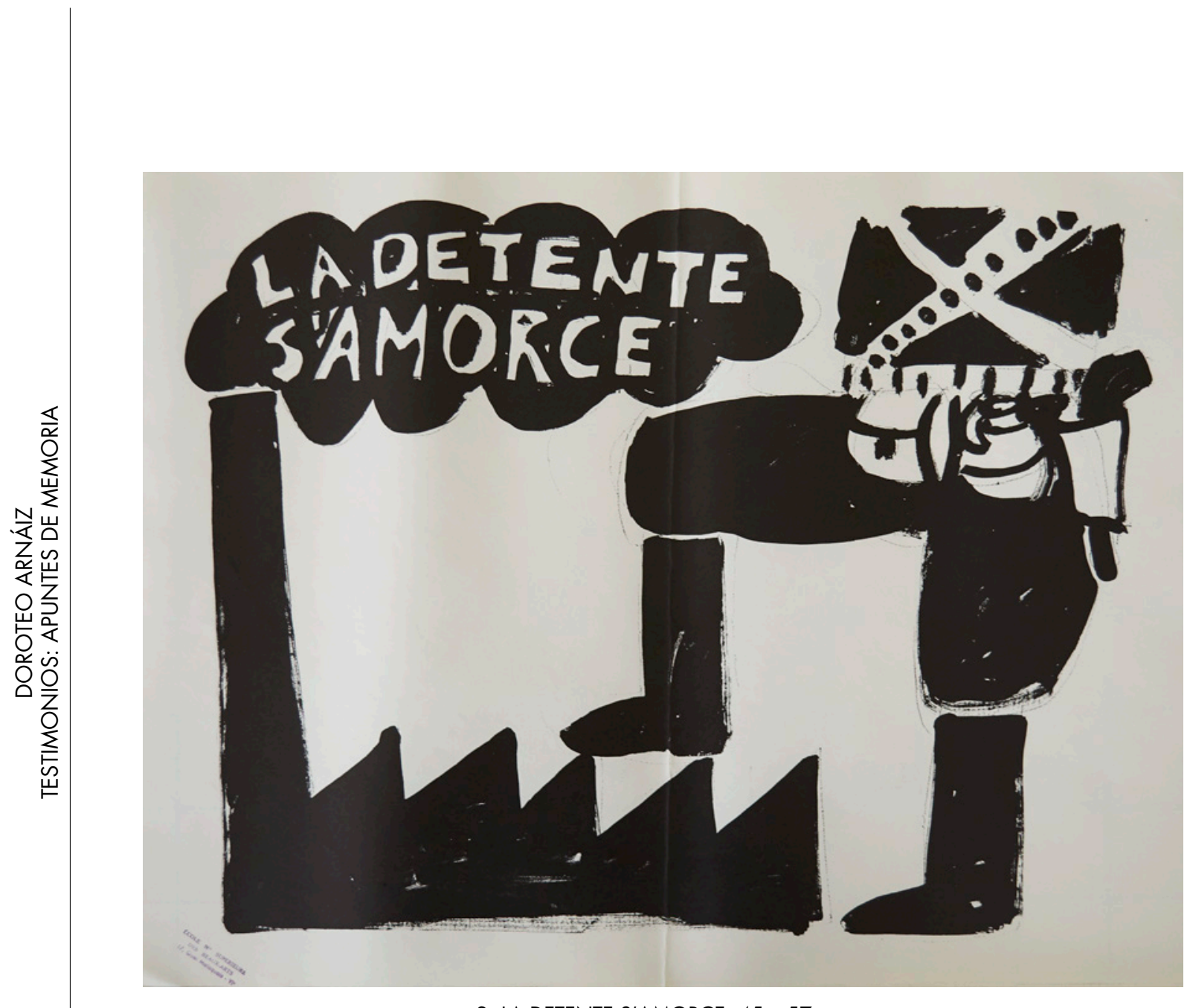

2. LA DETENTE S'AMORCE, $45 \times 57 \mathrm{~cm}$. 


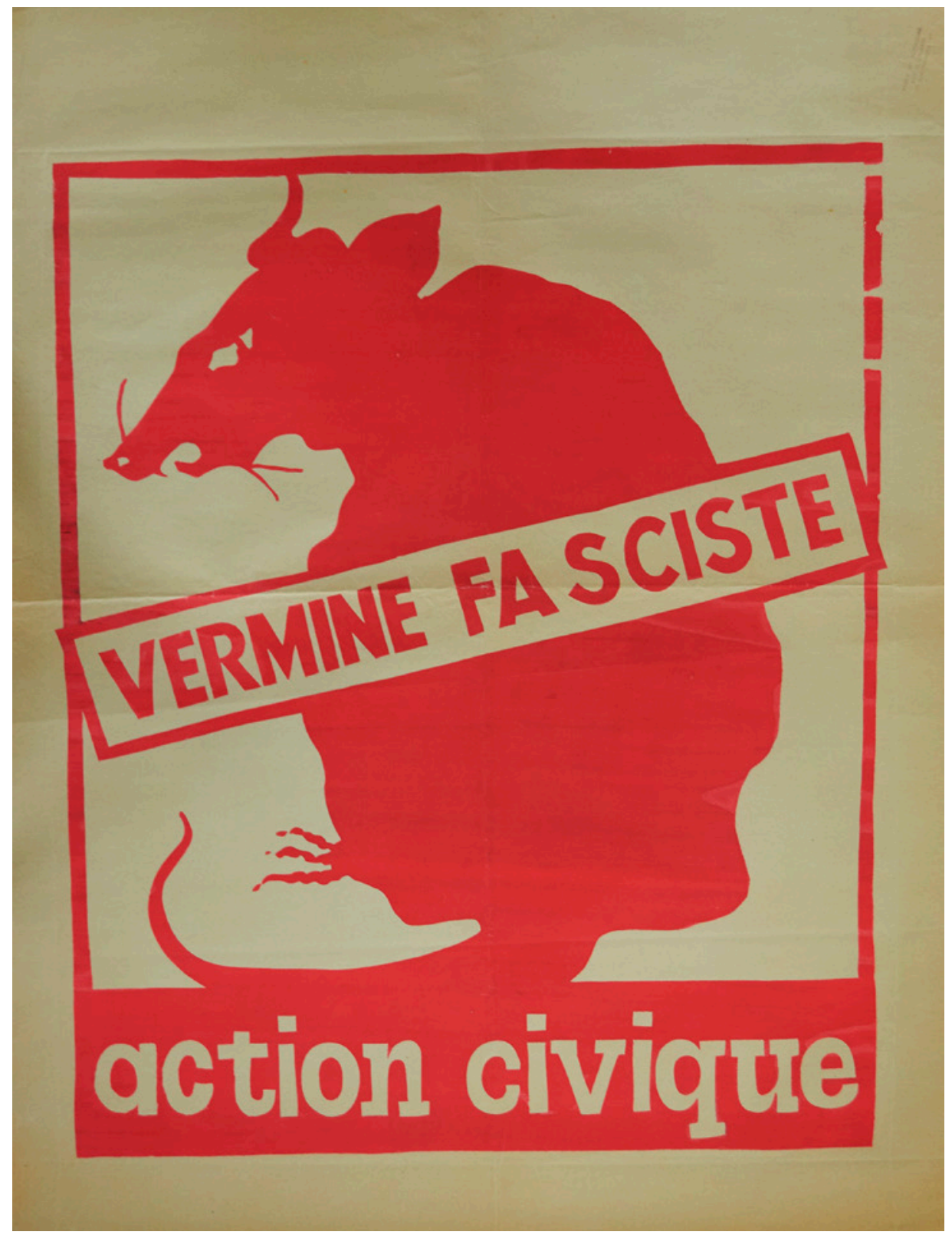

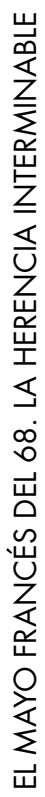

3. VERMINE FASCISTE, ACTION CIVIQUE, $80 \times 60 \mathrm{~cm}$. 


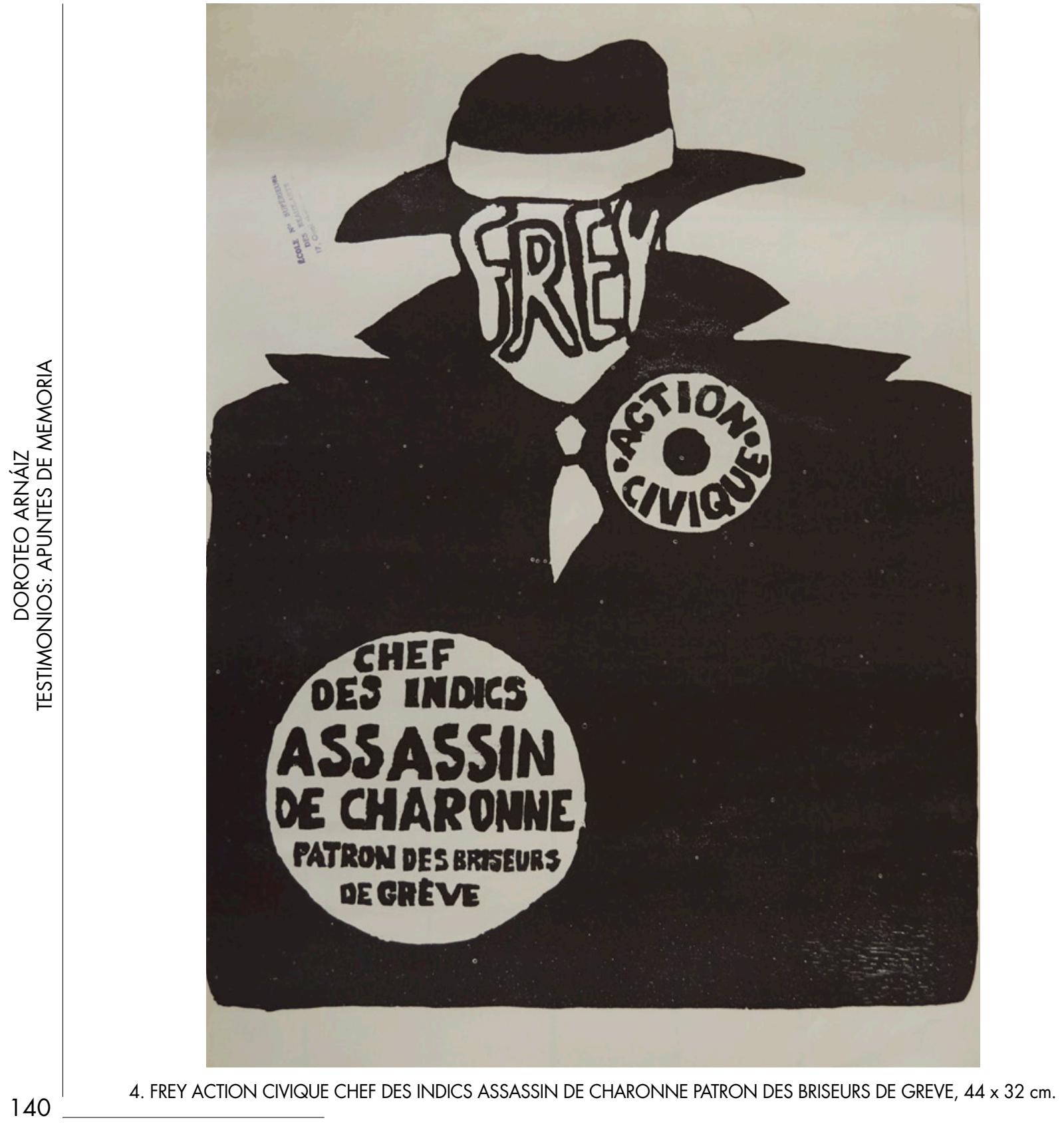

Dossiers Feministes, 24, 2018, 125-144 - ISSN: $1139-1219$ - DOI: http://dx.doi.org/10.6035/Dossiers.2018.24.9 


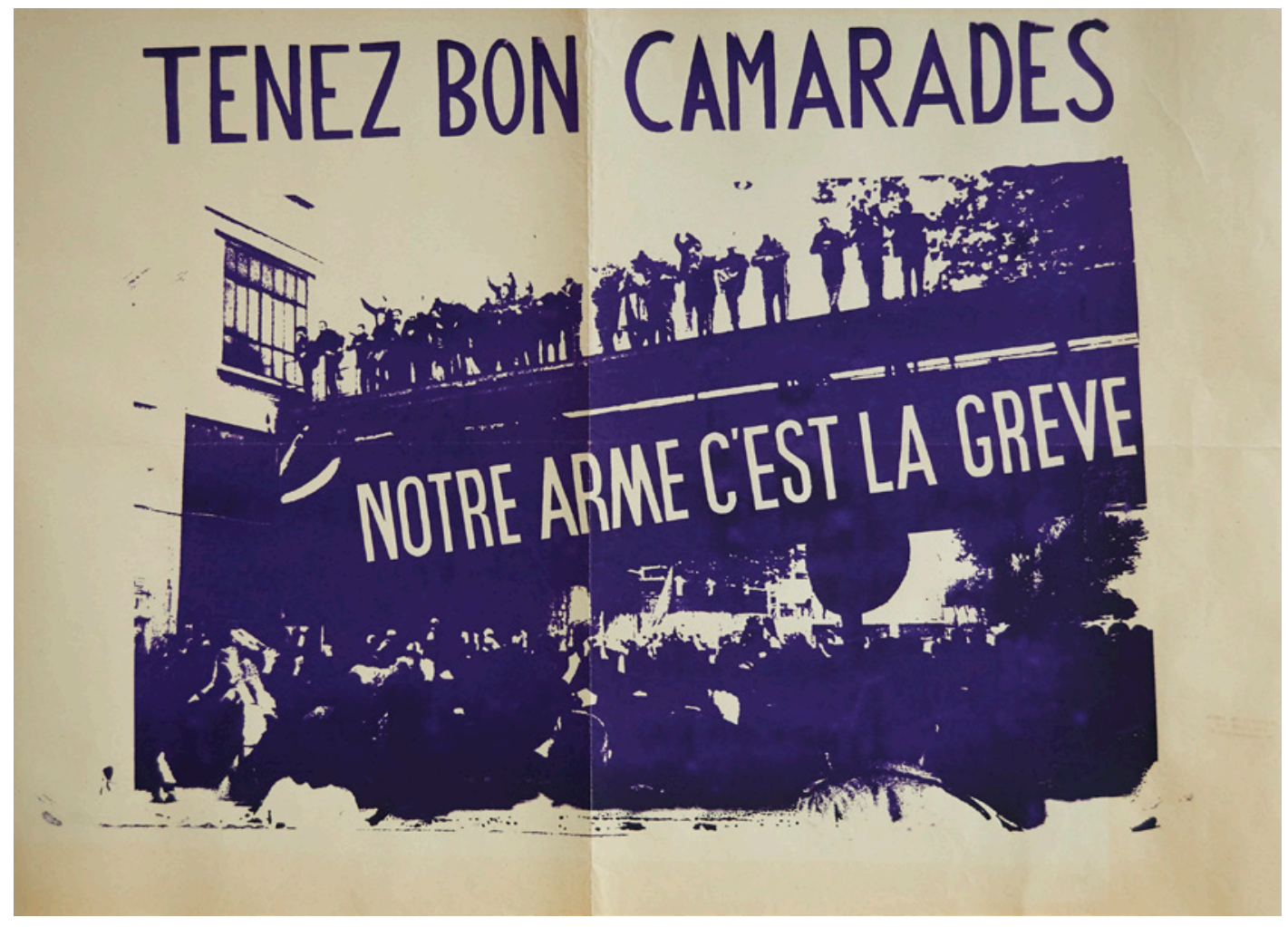

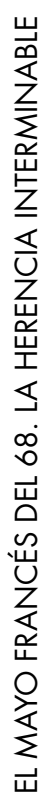

5. TENEZ BON CAMARADES NOTRE ARME C'EST LA GRÈVE, $56 \times 76 \mathrm{~cm}$. 


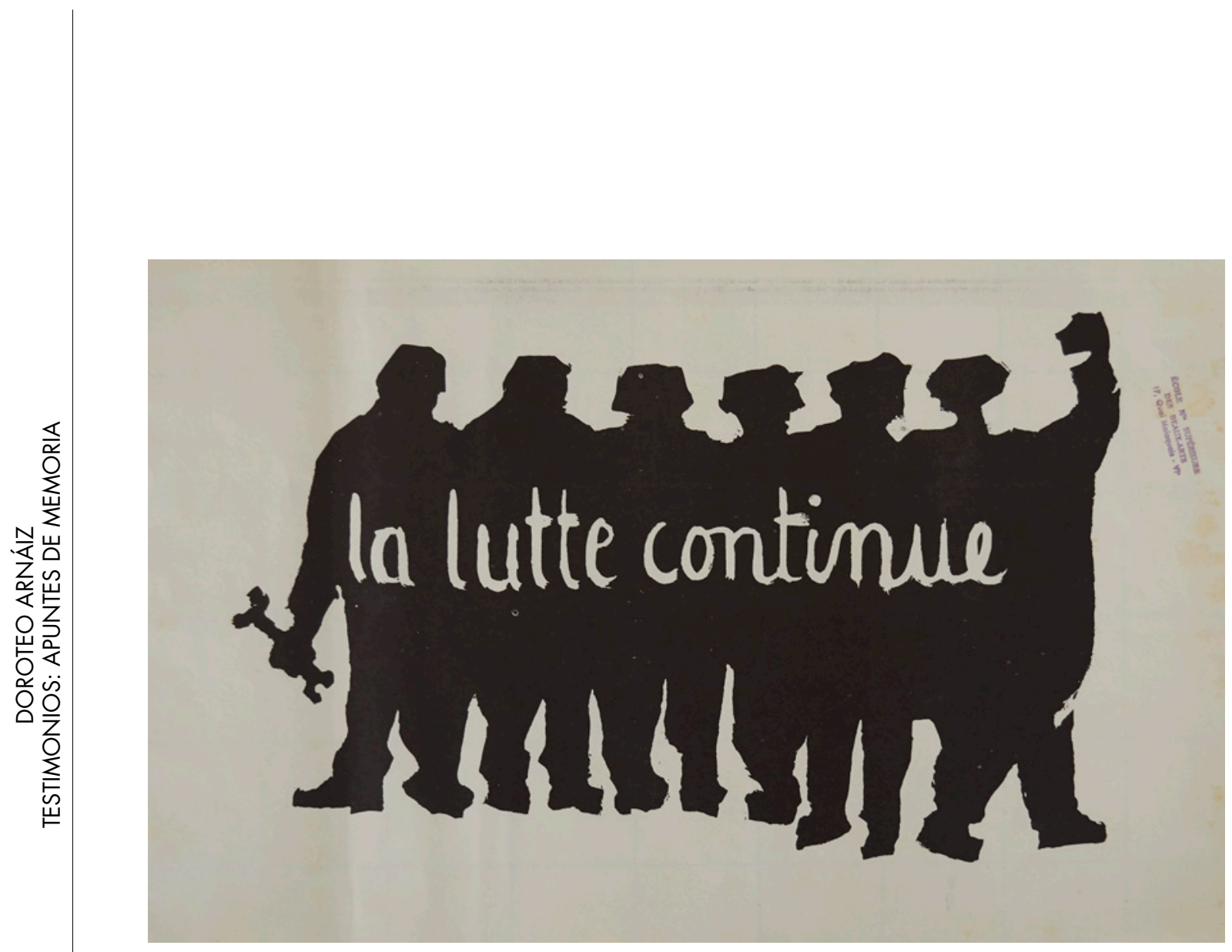

6. LA LUTTE CONTINUE, $28 \times 44 \mathrm{~cm}$. 


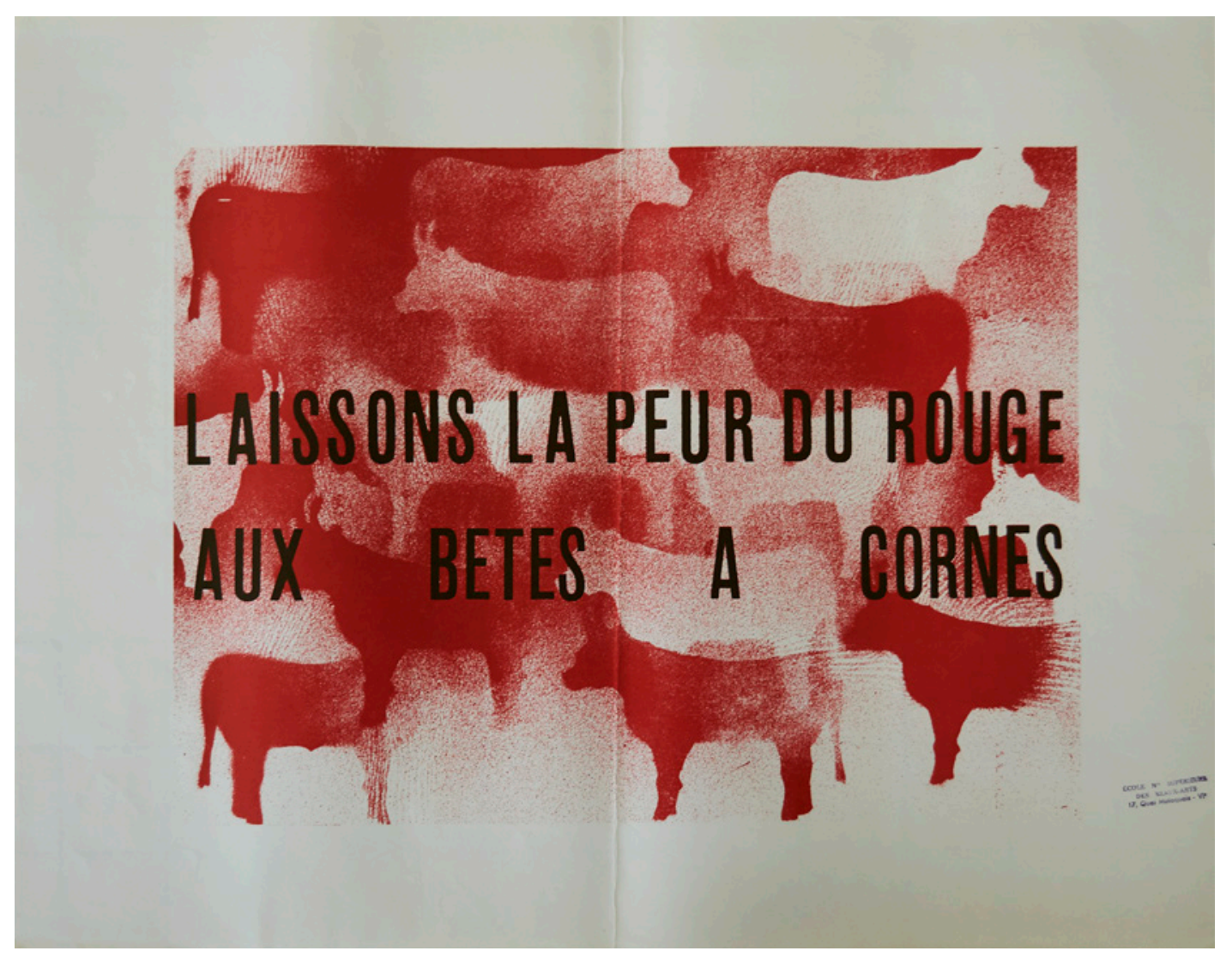

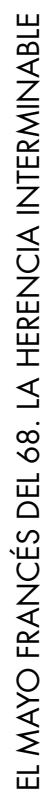

7. LAISSONS LA PEUR DU ROUGE AUX BETES A CORNES, $57 \times 45 \mathrm{~cm}$. 


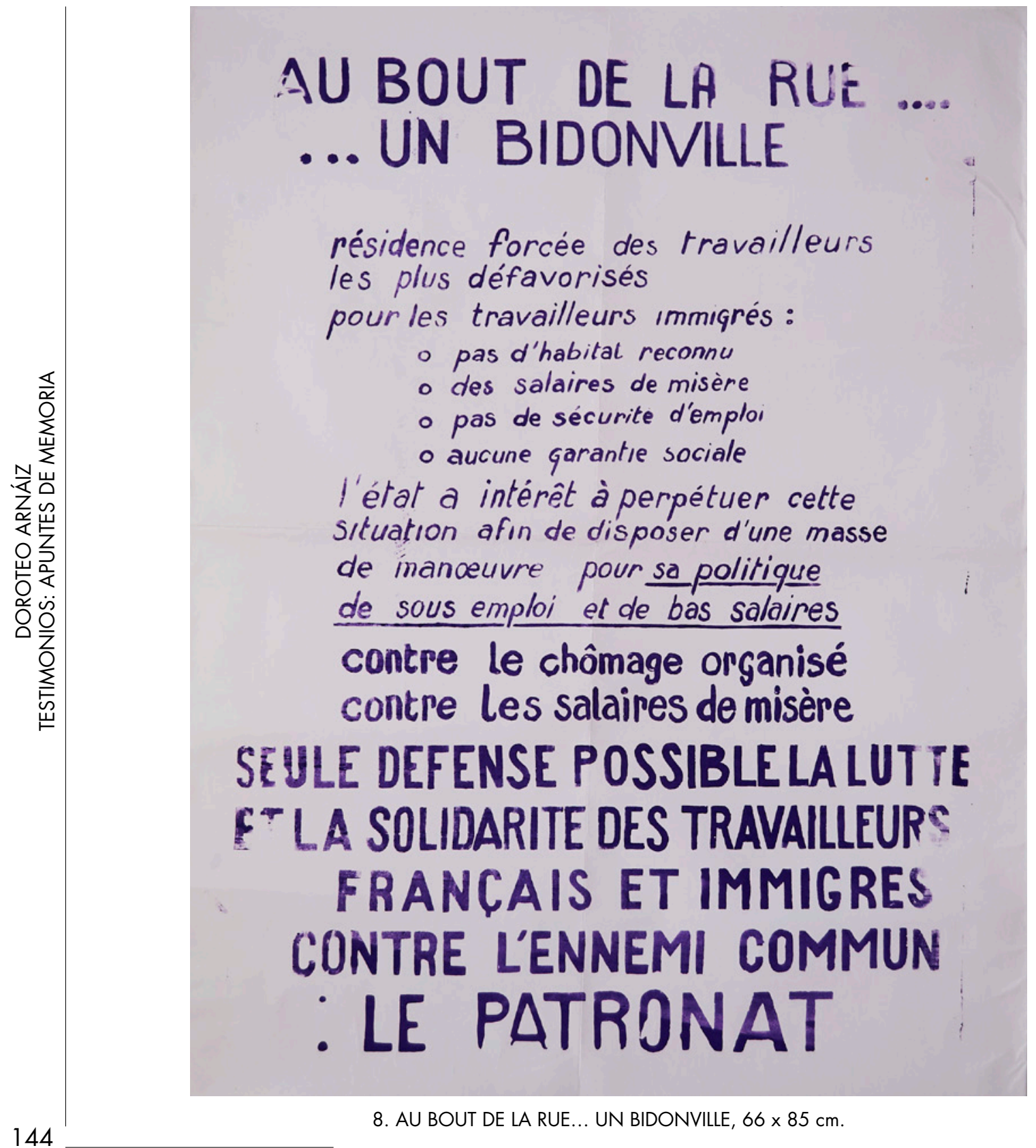

Dossiers Feministes, 24, 2018, 125-144 - ISSN: $1139-1219$ - DOI: http://dx.doi.org/10.6035/Dossiers.2018.24.9 